'Sección Inmunología Hospital

Clínico Universidad de Chile Departamento de Medicina Norte Facultad de Medicina Universidad de Chile. Santiago, Chile.

Trabajo no recibió financiamiento. La autora declara no tener conflicto de intereses.

Recibido el 9 de octubre de 2018, aceptado el 8 de octubre de 2019.

Correspondencia a:

Dra. Cecilia Sepúlveda C.

Sección Inmunología Hospital Clínico Universidad de Chile. Av. Santos Dumont 999,

$5^{\circ}$ piso Sector $\mathrm{E}$, Independencia. Santiago, Chile. dra.sepulveda@gmail.com

\section{Las mujeres en la medicina chilena: Desde los tiempos de Eloísa Díaz a la actualidad}

\author{
CECILIA SEPÚLVEDA C. ${ }^{1}$
}

\section{The presence of women in Chilean medicine: from the times of Eloísa Díaz to this day}

Though the presence of many women in medicine goes unnoticed today, their incorporation to the field was slow and difficult. It took until the end of the nineteenth century for women to be finally allowed to study at the University in Chile, being Eloisa Diaz the first woman to become a medical doctor in 1887. In that century, only six women became medical doctors. However, throughout the twentieth century, this small proportion of women in medicine increased at a similar rate as tuition did in the schools of medicine, especially from the 1960's when limited quotas for women were abolished. At present, women doctors constitute $40 \%$ of the total pool of medical doctors in Chile, being present in all specialties, though preferably found as general practitioners, family doctors and pediatricians. While many women have stood out in academia and in politics, they have also successfully reached high positions in scientific societies (and other offices such in the Health Ministry, and even the country's presidency) their participation is still limited in these areas. It is only fair to conclude that the outstanding participation that women in Chilean medicine enjoy today is not solely due to their long-lasting efforts, but more importantly, to their tireless struggle to overcome prejudice, discrimination and misunderstanding; the latter being especially represented by pioneer women of medicine.

(Rev Med Chile 2019; 147: 1184-1189)

Key words: Chile; Schools, Medical; History of Medicine; Physicians, Women.
CGT edado estaba para la mujer chilena franquear el umbral sagrado del augusto templo de la ciencia. La ley se oponía a ello cerrándole el paso que conducía a las aulas oficiales en las diversas gradaciones de la enseñanza secundaria y superior. La preocupación social que alguien con epiteto duro, pero indudablemente justo tildaría de añejo, se lo prohibía amenazándola con el duro ceño de su solemne encono y hasta con el cruel dictado de la reprobación condenatoria...". Con estas palabras, que reflejan los sentimientos y actitudes de la sociedad de la época, se graduaba la primera mujer chilena que recibía el título de médica, en enero de 1887, en la Universidad de Chile. Eloísa Díaz fue la primera mujer que obtuvo grados académicos y un título profesional universitario en Chile y Latinoamérica ${ }^{1}$.

Ella encabeza el grupo de seis médicas tituladas en el país en el siglo XIX, cuando las puertas de la Universidad se abrieron para las mujeres, luego que en 1877 se dictara el Decreto Amunátegui, que en lo esencial estableció: "las mujeres deben ser admitidas a rendir exámenes para obtener títulos profesionales, con tal que se sometan, para ello, a las mismas disposiciones a que están sujetos los hombres"2. 
El objetivo de este artículo es contribuir a visibilizar la presencia y aporte de las mujeres en la medicina en Chile, desde sus comienzos hasta nuestros días. La ausencia de mujeres en esta área ha sido una manifestación más de la discriminación general contra la mujer en la sociedad chilena y si bien hoy su presencia es importante, y pese a todo lo avanzado, persisten brechas de género que dificultan su acceso a ciertas especialidades, así como a cargos directivos y de liderazgo.

\section{Las primeras mujeres en la Escuela de Medicina de la Universidad de Chile}

Aquella época se caracterizó porque existían escuelas primarias públicas tanto para hombres como para mujeres, por separado, y también algunos establecimientos privados. Para efectuar estudios de enseñanza secundaria, las niñas debían optar por los escasos colegios privados que existían en el país, ya que la educación secundaria era solo para hombres. Según la legislación vigente entonces, para que los estudios secundarios fueran válidos para ingresar a la universidad, debían rendirse exámenes ante comisiones examinadoras de la Universidad de Chile. Las alumnas de colegios particulares no tenían ese derecho, por tanto, no tenían la posibilidad de acceder a la educación superior ${ }^{3}$.

Eloísa Díaz tenía 15 años cuando rindió dichas pruebas en 1881, causando gran impacto en el país. Es así como el 12 de abril de ese año, los periódicos publicaron la siguiente noticia: "El Claustro Universitario presentaba anoche una animación que no es frecuente en ese angosto recinto de la ciencia. Por primera vez en Chile, figuraba entre las aspirantes al Bachillerato en Humanidades, una estudiante del sexo femenino y tanto la novedad del hecho como la curiosidad despertada entre los alumnos de la Sección Universitaria habían logrado atraer a una numerosa concurrencia a la sala de exámenes". Eloísa Díaz rindió un examen brillante, siendo aclamada por la concurrencia y felicitada por las autoridades universitarias y, al día siguiente de obtener el grado de Bachiller en Humanidades, se matriculó en la Escuela de Medicina de la Universidad de Chile, donde cursó brillantemente los seis años de estudio que exigía en ese entonces la carrera de medicina, recibiendo premios y honores por su excelente desempeño ${ }^{4}$.
Las otras mujeres médicas en aquel tiempo fueron Ernestina Pérez (graduada una semana después que Eloísa Díaz, en 1887), Eva Quezada (1894), Pilar Pérez (1894), Ema Cosio (1898) y Elvira Higueras (1889). Resulta interesante señalar que, en el mismo año en que Eloísa Díaz y Ernestina Pérez se graduaban de médicas en Chile, por primera vez se admitían, con mucha dificultad, mujeres en la carrera de medicina en Francia y en Prusia se dictaba una orden que prohibía la admisión de mujeres en las universidades del imperio ${ }^{5}$.

Ernestina Pérez, luego de titulada, obtuvo una beca para especializarse en ginecología en Alemania, cuando en dicho país no se admitían mujeres en medicina, debiendo convencer a las autoridades universitarias que la recibiesen y escuchar las clases detrás de un biombo ${ }^{6}$.

El trato que se dio a estas primeras estudiantes dentro de la Universidad en Chile, no fue igual en todos los casos. Crónicas de la época relatan que éstas fueron bien recibidas por sus compañeros, mientras que otras describen que ambas debían asistir a clases con sus madres y que "eran miradas en menos, costándoles ganarse el respeto de sus profesores y compañeros". Ya tituladas, estas dos primeras médicas destacaron por su interés por los problemas de salud de mujeres y niños, por sus aportes a la medicina social y a la docencia, y con el tiempo recibieron el reconocimiento de sus pares, de la sociedad y del gobierno de Chile ${ }^{7}$.

$\mathrm{Su}$ gran mérito, reside, a mi juicio, en que ellas abrieron a la mujer chilena, la gran puerta de la educación superior, al mismo tiempo de haber contribuido a que otras pudieran acceder también a ella.

La figura cumbre de este siglo, sin duda fue Eloísa Díaz. En 1887 fue nombrada por el gobierno Médica Inspectora de las Escuelas Públicas de Santiago, cargo que desempeñó por más de 30 años. A su iniciativa se debe la creación del servicio médico y dental en las escuelas, la fundación de jardines infantiles, colonias escolares de vacaciones, policlínicos para atender a los alumnos de menores recursos y desvalidos, vacunación obligatoria, cantinas escolares, y muchos otros logros. Reconocida internacionalmente, fue nombrada "Hija Ilustre de América en el Congreso Científico Internacional de Medicina en Buenos Aires, en $1910^{8}$. 


\section{Las mujeres médicas en Chile según las estadísticas}

Hasta 1920 solo un número reducido de mujeres se había graduado de médica; posteriormente, en forma paulatina, fue aumentando progresivamente el número de graduadas, hasta llegar a constituir un número importante hacia fines del siglo.

Esta lenta incorporación en la profesión médica no solo se relaciona con el casi nulo acceso de la mujer a la educación superior en esos años, sino que también, a que las vacantes en las escuelas de medicina estuvieron restringidas o prohibidas para las mujeres hasta avanzado el siglo $\mathrm{XX}^{5}$.

Es de interés señalar que de acuerdo al censo de 1907, la población total del país era de 3.249.279, siendo las mujeres 1.625.058. De ellas, 361.012 trabajaban remuneradamente: 126.666 eran costureras; 67.682 se desempeñaban como empleadas domésticas; 62.977 como lavanderas y 24.963 eran artesanas. El número de mujeres profesionales era insignificante. Solo 3 eran abogadas, 7 médicas, 10 dentistas y 10 farmacéuticas, mientras que había 3.980 mujeres dedicadas a la enseñanza y 1.070 matronas 5 .

En 1920, de una población de mujeres de 1.887.972, trabajan fuera de la casa 346.836 , y el número de mujeres que estudia es de 150.154, aunque de ellas muy pocas logran llegar a la enseñanza superior. De un total de 800 médicos, se reportan 33 mujeres.

En 1940, la población total era de 5.023.539, siendo de 2.533.613 la femenina. En veinte años se había duplicado el número de mujeres que estudiaba y las mujeres profesionales aumentaron, entre ellas las médicas que llegan a 263.

Según el censo de 1952, la población femenina chilena era de 3.020.437, de un total de 5.932.995. Aumentan las mujeres que estudian, que alcanzan a 489.141, pero de ellas, solo 6.327 llegan a la universidad. Esto es menos del $2 \%$ del total de las mujeres estudiantes 9 .

A fines del siglo XX, el censo de población de 1982, registró 6.748 hombres médicos y 2.128 mujeres médicas en el país. Esto es, uno de cada cuatro médicos en Chile eran mujeres en esa época ${ }^{10}$.

En 1992, el Instituto Nacional de Estadísticas (INE) registra 9.689 médicos hombres y 4.168 mujeres. Ya uno de cada tres médicos en Chile es mujer, denotando el interés de las mujeres por esta profesión. Y en el año 2008, el INE informa que ejercen en el país 22.247 médicos, de los cuales 7.265 son mujeres, manteniéndose la proporción de una mujer por cada tres médicos hombres ${ }^{11,12}$.

Esta situación cambia drásticamente en la última década. A diciembre de 2016, de 42.623 médicos en el Registro Nacional de Prestadores Individuales de la Superintendencia de Salud, casi $40 \%$ son mujeres, destacando que $47 \%$ de los médicos menores de 35 años son mujeres ${ }^{13}$.

\section{La matrícula de mujeres en medicina}

Desde su creación, en 1842, la Universidad de Chile fue la única institución de educación superior del país donde se impartían los estudios de medicina; $y$, si bien la universidad debió aceptar mujeres en la Escuela de Medicina (según lo establecido por el decreto Amunategui de 1887), las vacantes para ellas estuvieron restringidas a $10 \%$ en cada promoción. Esto hasta la década de 1960-699.

Hasta 1990, la carrera era impartida en seis universidades. Estas fueron aumentando en el tiempo, de tal modo que hoy día se dicta en 26 universidades, expandiendo las vacantes desde 559 en ese año, a 2.450 en 2018. En 2007, la matrícula total en medicina es de 10.168 y llega a 15.807 en 2018. En dicho período, la matrícula total de mujeres se duplicó desde 4.616 en el año 2007 a 8.081 en el 2018 ; esto es, aumentó de $45,4 \%$ a $51,1 \%$ del total ${ }^{14,15}$.

Recién en el año 1924, la Universidad de Concepción abrió la carrera de medicina, también con vacantes restringidas para las mujeres, y solo a partir del año 1953 se permitió el ingreso de mujeres en la Pontificia Universidad Católica de Chile (carrera creada en 1930), igualmente restringido. Las primeras admitidas fueron Elena Castro, Catalina Maggiolo y Carmen Wilson, todas tituladas en los primeros lugares de su promoción ${ }^{16,17}$.

\section{El ejercicio de la medicina y las especialidades}

Entre 1910 y 1960 muchas mujeres médicas hicieron importantes aportes a la medicina, entre ellas Amparo Arcaya, primera psiquiatra infantil, Juana Ardiman, que se especializó en pacientes quemados y organizó el banco para quemados en 
el Hospital San Luis, y la ginecóloga Juana Díaz, que fue socia fundadora de la Asociación Médica Femenina de Chile y del Centro contra el Cáncer, Eleanira González fue la primera cirujana en Chile y organizó la Escuela de Enfermeras de la cual fue su directora, María Hamuy, psiquiatra, dirigió el Hospital Psiquiátrico y logró que se construyera el edificio que actualmente ocupa ese hospital en Avenida La Paz en Santiago y Cora Mayers, quien trabajó en Gotas de leche, dirigió la Escuela de Enfermeras, creó el Departamento de Educación Sanitaria de la Dirección General de Sanidad, y sucedió a Eloísa Díaz en el Consejo de Instrucción Primaria, entre muchas otras ${ }^{5,11}$.

En la actualidad se observa la presencia femenina en todas las especialidades y subespecialidades médicas, algunas especialidades aún no cuentan con una importante presencia femenina, como cirugía $^{18}$, traumatología, urología y otras. Por el contrario, muchas médicas se han dedicado preferentemente a la medicina general, medicina familiar y a la pediatría ${ }^{19}$.

Hasta hace pocos años, si bien muchísimas médicas se desempeñaban en la salud pública a lo largo del país, era poco frecuente encontrar a una mujer como directora de hospital o en puestos de alta responsabilidad en el sistema de salud pública. Recién en 1998, por primera vez, una mujer fue nombrada directora de un servicio de salud, la Dra. María Luisa Cayuela. Esta tendencia se rompe definitivamente con el nombramiento de la Dra. Michelle Bachelet como ministra de salud en el año 2000, seguida posteriormente por Soledad Barría, Helia Molina y Carmen Castillo. Son 4 ministras entre más de 60 que han ocupado este cargo desde 1924 hasta la actualidad ${ }^{20}$.

Desde entonces es más frecuente encontrar mujeres médicas, ya sea en cargos de alta responsabilidad en los hospitales o en el Ministerio de Salud. Así, muchas mujeres son actualmente directoras de hospital y jefas de servicio a lo largo de Chile.

\section{La mujer médica en la academia}

Muchas mujeres médicas empezaron a destacar en la docencia de la medicina. La primera de ellas fue Victoria García, titulada en 1935, especializada en educación sanitaria, primera profesora extraordinaria de la Facultad de Medicina de la
Universidad de Chile en la Escuela de Salubridad y miembro del consejo. De las mujeres tituladas entre los años 1910 y 1960, podemos mencionar además a varias médicas destacadas en la academia. Entre ellas, Violeta Cerda, quien fuera ayudante de Cátedra de Otorrinolaringología de la Universidad de Chile; Berta Cristo, ayudante de Cátedra de Clínica Quirúrgica en el antiguo Hospital San Borja; a mediados del siglo pasado, Ema Dolci, pediatra, Amalia Ernst, ginecóloga y Olga Fisher, también ginecóloga, fueron también ayudantes de sus respectivas cátedras ${ }^{11}$.

La primera en estar a cargo de una cátedra fue Cora Mayers, en la Cátedra de Puericultura de la Escuela de Medicina de la Universidad de Chile; Marta Montenegro también tuvo un rol muy destacado en la docencia en la Facultad de Medicina de la Universidad de Chile; Obdulia Jiménez, neuróloga infantil, fue profesora auxiliar de la Escuela de Medicina de la Universidad de Concepción; son solo algunas de las numerosas médicas que efectuaron en esos años enormes aportes a la docencia, destacando por su compromiso y gran dedicación ${ }^{21}$.

Avanzado el siglo XX, muchas otras mujeres médicas irrumpieron en la academia, como Ester Mateluna, pediatra y quien fuera directora de la Escuela de Medicina de la Universidad de Chile, Teresa Pinto, neurofisióloga, una de las primeras mujeres en llegar a ser investigadora independiente en esa misma facultad. Podemos también mencionar a las salubristas profesoras de la Escuela de Salud Pública de la Universidad de Chile, Tegualda Monreal, Aída Kirschbaum y Ana María Kaempffer, a Cristina Palma y Ximena Berríos de la Pontificia Universidad Católica de Chile ${ }^{22}$. Son solo algunas de muchas mujeres médicas que lograron el reconocimiento de sus pares y asumen responsabilidades cada vez mayores a partir de la segunda mitad del siglo XX.

Pero pese a que la mujer médica cada vez ha ido ganando más espacios en diferentes ámbitos del quehacer académico, pocas llegan a ser profesoras titulares o investigadoras independientes. Escasas son quienes llegan a ser directoras de departamentos universitarios y decanas y nunca una mujer elegida por sus pares ha sido rectora. La autora de este artículo fue la primera vicerrectora académica de la Universidad de Chile (2002-2006) y decana elegida de la Facultad de Medicina de la Universidad de Chile (2002-2006 y 2006-2010). 
En la Academia de Medicina, desde su creación en el año 1964, nunca una mujer ha sido presidenta de esta entidad y tres han sido secretaria o tesorera. De los miembros de número de esta academia, la primera mujer en este grupo es Marta Velasco, elegida por sus pares para ocupar un sillón, en 1992. Posteriormente se han incorporado como miembros de número, Gloria López, Colomba Norero, Sylvia Segovia, Gloria Valdés, Mireya Lechat, Valeria Prado, Cecilia Albala y María Eugenia Pinto. Son ocho mujeres entre 77 miembros a lo largo del tiempo ${ }^{23}$. Virginia Gómez y Angela Muñoz figuran en el registro de los 39 socios fundadores de la Sociedad Médica de Concepción, en $1924^{15}$.

Desde los tiempos de Eloísa Díaz y Cora Mayers, fundadoras de la Sociedad de Pediatría, solo cuatro mujeres han sido presidentas de esta sociedad, siendo Helia Molina la primera en presidirla en 1983 (y luego ministra de salud en 2014).

La Sociedad de Obstetricia y Ginecología, también ha sido presidida por dos mujeres: Sylvia Segovia en 1980 y Mercedes Ruiz en 2002. En la Sociedad de Cirujanos, hasta ahora solo ha habido una mujer presidenta: la Dra. Yamile Camacho en $2010^{18}$. Otro hito importante fue la elección de Paulina Baquedano en 2015 como presidenta de la Sociedad Chilena de Urología, una sociedad formada mayoritariamente por hombres.

En la actualidad es frecuente que muchas mujeres formen parte de la directiva de las sociedades científicas, como secretarias o tesoreras, pero no así como presidentas. Solo 6 de 28 sociedades científicas médicas son presididas por una mujer en la actualidad ${ }^{24}$.

\section{Destacadas en la política y en los gremios}

En el ámbito gremial, a mediados de 2017, Izkia Siches asumió la presidencia de un gremio que por más de 71 años había sido encabezado por hombres, convirtiéndose en la primera mujer en presidir el Colegio Médico de Chile AG elegida por sus pares. También fue la primera mujer presidenta del Regional Santiago ${ }^{25}$.

También mujeres médicas se han destacado en la política en nuestro país. Entre ellas María Elena Carrera, quien fue la primera médica senadora en los años 60 del siglo pasado, Fanny Pollarolo, también diputada, a fines del siglo XX. En la ac- tualidad la intendenta de la Región Metropolitana Karla Rubilar, ex diputada; la senadora Jacqueline van Rysselberghe, la diputada Marcela Hernando, y la ex alcaldesa Vicky Barahona. En otro ámbito, destaca Marisol Prado, primera mujer presidenta de la centenaria Federación de Estudiantes de la Universidad de Chile (FECH) en 1997 ${ }^{11,26}$.

Sin duda, la elección de Michelle Bachelet como presidenta de la República en 2005 es un hito importante que refuerza la percepción de que las médicas están preparadas para asumir cualquier rol, tanto en el ámbito público como privado.

Al finalizar esta revisión, podemos destacar que si bien la incorporación de la mujer en la medicina chilena fue lenta y difícil en sus inicios, en la actualidad las médicas constituyen una importante proporción del cuerpo médico del país y el continuo incremento femenino en la matrícula de la carrera en las últimas décadas sugiere que esta presencia continuará aumentando. Las mujeres se han incorporado en prácticamente todos los ámbitos y especialidades de la medicina, aunque su acceso a cargos y responsabilidades de envergadura aún es limitado.

A modo de conclusión, se puede señalar que la destacada participación que tienen hoy las mujeres en la medicina chilena se debe a la larga y no pocas veces fatigosa lucha que dieron venciendo prejuicios, discriminaciones e incomprensiones, destacando en ella particularmente las mujeres pioneras de la medicina, cuyos aportes constituyen un importante legado que perdura hasta hoy.

\section{Referencias}

1. Díaz E. Extracto de la Introducción de su Memoria de Prueba para optar al grado de Licenciado de la Facultad de Medicina y Farmacia, leída el 25 de diciembre de 1886, en la Universidad de Chile. Disponible en web http://www.revistadeeducacion.cl/eloisa-diaz-insunza-el-derecho-de-la-mujer-a-la-educacion-universitaria/

2. Decreto S/N 04-MAR-1877. Ministerio de Justicia, Culto e Instrucción Pública, Biblioteca del Congreso Nacional de Chile. Disponible en web https://www.archivonacional.gob.cl/616/w3-article-8046.html?_noredirect=1.

3. Sánchez K. El ingreso de la mujer chilena a la Universidad y los cambios en la costumbre por medio de la Ley 1872-1877. Historia (Santiago) [online]. 2006, vol.39, n.2 [citado el 29 de agosto de 2018], pp. 497-529. 
4. http://www.revistadeeducacion.cl/eloisa-diaz-insunza-el-derecho-de-la-mujer-a-la-educacion-universitaria/

5. Klimpel F. La mujer chilena (el aporte femenino al Progreso de Chile) 1910-1960. Editorial Andrés Bello; 1962. p. 158-60.

6. http://www.revistadeeducacion.cl/ernestina-perez-barahona-el-valiente-itinerario-de-una-medico-cirujano/

7. www.bibliotecaminsal.cl/wp/wp-content/uploads/.../ Biografía-Dra.-Eloisa-Diaz.pdf.

8. Higiene Escolar. Eloísa Díaz. Biblioteca Fundamentos de la Construcción de Chile. Cámara Chilena de la Construcción. Pontificia Universidad Católica de Chile. Biblioteca Nacional. Septiembre de 2011. Hitos en la Biografía de Eloísa Díaz. Pág. xLii-xLiii Disponible en: http://biblioteca.cchc.cl/datafiles/25711-2.pdf.

9. Mujeres y profesiones universitarias (1900-1950): Grupo diverso de mujeres profesionales http://www.memoriachilena.cl/602/w3-article-96245.html.

10. XII Censo General de Población y de Vivienda. Servicio Nacional de Estadística y Censos. República de Chile. 1982. Disponible en web http://www.memoriachilena. gob.cl/archivos2/pdfs/MC0055466.pdf.

11. Sepúlveda C. Cap 3. Las profesiones y los oficios. Las mujeres chilenas en la medicina. En: Mujeres chilenas. Fragmentos de una historia. Compiladora Sonia Montecino. Editorial Catalonia; 2008. p. 165-72.

12. Estadísticas de Salud. Compendio estadístico INE. 2009. p. 227. Disponible enhttp://historico.ine.cl/ canales/menu/publicaciones/compendio_estadistico/ pdf/2009/1_6_estadisticas_de_salud.pdf.

13. Registro Nacional de técnicos y profesionales de salud con título habilitante. Superintendencia de Salud. Diciembre de 2016. http://www.supersalud.gob.cl/servicios/669/w3-article-5587.html.

14. Matrícula histórica 2007-2018 del SIES, Mineduc. Disponible en:http//www.mi futuro.cl/index.php/bases-de-datos/matriculados.

15. Bastías G, Marshall G, Zúñiga D, Mena B. Número de médicos en Chile: estimaciones, proyecciones y com- paración internacional. Rev Med Chile 2000; 128 (10): 1167-76.

16. Wilhelm O. Historia de la medicina penquista. Discurso sesión solemne por el septuagésimo quinto aniversario de la Fundación de la Sociedad Médica de Concepción. 5 de junio de 1962. p. 125 Disponible en web http://www.bibliotecaminsal.cl/wp/wp-content/ uploads/2013/05/anales_ano4_vol1_p103.pdf.

17. Vargas JE, Chuaqui B, Duarte I. Médicos de Ciencia y Conciencia. La Escuela de Medicina de la Pontificia Universidad Católica de Chile. Ediciones Universidad Católica de Chile; 2005. p.141.

18. Espinoza R, Onetto C, Lombardi JJ, Espinoza JP. La incorporación de la mujer en la cirugía chilena. Rev Chilena de Cirugía 2012; 64 (5): 487-91.

19. Informe sobre brechas de personal de salud por servicio de salud. Ministerio de Salud. Subsecretaría de redes Asistenciales. División de Gestión y Desarrollo de las Personas. Departamento de Planificación y Control de Gestión de RHS. 2017. Disponible en web https://www. minsal.cl/wp-content/uploads/2015/08/Informe-Brechas-RHS-en-Sector-P\%C3\%BAblico_Abril2017.pdf.

20. Histórico de Ministros - Ministerio de Salud - Disponible en web https://www.minsal.cl/historial-de-ministros-de-salud/

21. Stuven AM, Fernandois J. Historia de las mujeres en Chile. Tomo 2. Zárate MS. Al cuidado femenino, mujeres y profesiones sanitarias, Chile,1889-1950. Publicado por Penguin Random House Grupo Editorial Chile. 2014.

22. Kirschbaum A. Las mujeres en la medicina en Chile. Rev Chil Salud Pública 2000; 4 (2-3): 145-50.

23. Nómina de miembros Academia de Medicina http:// www.academiachilenademedicina.cl/cont.php?id=158.

24. Sociedades Científicas Médicas. https://asocimed.cl/site/

25. Presidencia Colegio Médico de Chile http://www.colegiomedico.cl/?page_id=1502.

26. https://universitarios.cl/2013/06/16/archivo-fech-rescate-de-la-historia-estudiantil/ 